\title{
Variable optical attenuator with a polymer-stabilized dual-frequency liquid crystal
}

\author{
Yung-Hsun Wu, Xiao Liang, Yan-Qing Lu, Fang Du, Yi-Hsin Lin, and Shin-Tson Wu
}

\begin{abstract}
A transmission-type variable optical attenuator (VOA) based on a polymer-stabilized dual-frequency liquid crystal (PSDFLC) is demonstrated at $\lambda=1.55 \mu \mathrm{m}$. The VOA is highly transparent in the voltageoff state but scatters light in the voltage-on state. By using a birefringent beam displacer incorporated with half-wave plates, we can obtain a VOA that is polarization independent and that exhibits a $31 \mathrm{~dB}$ dynamic range. The polymer networks and dual-frequency effect together reduce the response time (rise + decay) of a $16 \mu \mathrm{m}$ PSDFLC cell to $30 \mathrm{~ms}$ at room temperature and at a voltage of $24 \mathrm{~V}_{\mathrm{rms}}$. (C) 2005 Optical Society of America

OCIS codes: $230.3720,160.5470$.
\end{abstract}

\section{Introduction}

A polymer-stabilized liquid crystal ${ }^{1-3}$ (PSLC) is a fundamentally interesting and practically useful material system. Both anisotropic scattering ${ }^{4}$ and scattering-free ${ }^{5}$ PSLC systems have been developed, depending on the polymer concentration and operating wavelength. The anisotropic light-scattering PSLC is useful for amplitude modulation because it has, e.g., a reflective display, switchable polarizer, and variable optical attenuator (VOA). ${ }^{6-10}$ On the other hand, the scattering-free PSLC is particularly attractive for phase modulation in the near-infrared region. ${ }^{5}$ In spite of the need for amplitude or phase modulation, a fast response time is a standard requirement. This is particularly problematic for VOAs operating in the near-infrared region $(\lambda=1.55 \mu \mathrm{m})$, where a larger cell gap is required, which results in a longer response time. To keep the response time short, a PSLC-based reflective VOA has been considered. For a $16 \mu \mathrm{m}$ reflective PSLC cell using a Merck E44-LC mixture, the measured response time is $\sim 30 \mathrm{~ms}$ and the dynamic range is $\sim 30 \mathrm{~dB}$ at room temperature. If we use the cell parameters in the transmissive mode, the dynamic range would be reduced to $\sim 15 \mathrm{~dB}$. If we want to keep the same dy-

The authors are with the College of Optics and Photonics, University of Central Florida, 4000 Central Florida Boulevard, Orlando, Florida 32816. S.-T. Wu's e-mail address is swu@mail.ucf.edu. Received 8 December 2004; accepted 8 February 2005. 0003-6935/05/204394-04\$15.00/0

(C) 2005 Optical Society of America namic range, then we need to double the cell gap, but the response time would be $4 \times$ slower.

In this paper we demonstrate a polarizationindependent transmissive VOA by using the polymerstabilized dual-frequency liquid crystal (PSDFLC) to obtain a large dynamic range $(31 \mathrm{~dB})$ while keeping a $30 \mathrm{~ms}$ frame time. The unique feature of a DFLC is that it exhibits a crossover frequency $\left(f_{c}\right){ }^{11,12}$ The dielectric anisotropy of the DFLC remains positive $(\Delta \varepsilon>0)$ when $f<f_{c}$ but changes to negative when $f>f_{c}$. For practical applications, the crossover frequency is usually in the $5-10 \mathrm{kHz}$ range. For the DFLC-based homogeneous cell, we use a lowfrequency $(\sim 1 \mathrm{kHz})$ electric field to drive the VOA and a high-frequency $(\sim 30 \mathrm{kHz})$ electric field to accelerate the relaxation process. Owing to the presence of electric field in both turn-on and turn-off processes, fast rise and decay times can be obtained simultaneously.

\section{Light Modulation Mechanism}

Figure 1 illustrates the light modulation mechanism of the PSDFLC cell. If a low-frequency $\left(f<f_{c}\right)$ voltage is applied to the cell, as shown in Fig. 1(a), the polymer networks tend to resist LC molecules from being reoriented by the electric field. As a result, microdomains are formed, which scatter the extraordinary ray because of the refractive-index mismatch between the DFLC and the polymer networks. The ordinary ray is not affected because of good index match with the polymer matrix. If we keep the ac voltage unchanged but switch the frequency to a high frequency $\left(f>f_{c}\right)$, the electric field exerts a torque to bring the LC directors back to their original homo- 


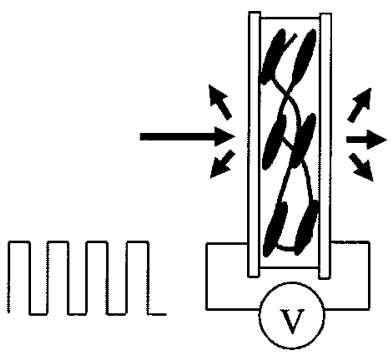

(a)

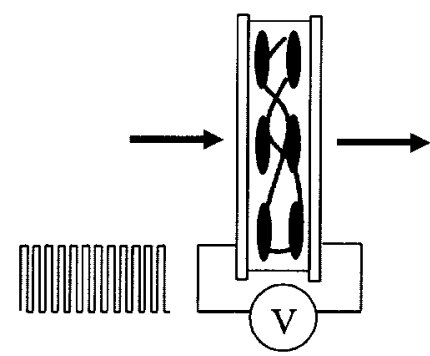

(b)
Fig. 1. Light modulation mechanism of a PSDFLC cell. (a) At low-frequency $(1 \mathrm{kHz})$, light scattering occurs for the extraordinary ray. (b) At high frequency $(30 \mathrm{kHz})$, the device is highly transparent.

geneous positions, as shown in Fig. 1(b). Thus the PSDFLC cell is highly transparent. The anisotropic light-scattering behavior of the PSDFLC cell is polarization dependent. For fiber-optic communication, it is highly desirable to have a VOA whose performance is independent of light polarization.

Because the PSDFLC cell attenuates only the extraordinary ray, we need to convert the incident unpolarized light into linearly polarized light. Figure 2 shows such a device configuration. The polarization beam displacer (BD) used in Fig. 2 is a $45^{\circ}$-cut birefringence crystal, e.g., $\mathrm{YVO}_{4}$ or calcite. Inside the beam displacer, the incident unpolarized light is separated into two components: ordinary ray (upper trace) and extraordinary ray (lower trace). The halfwave plate on the top part of the beam displacer transforms the ordinary ray into an extraordinary ray. The rubbing axis of the PSDFLC cell is positioned parallel to the polarization axis. The outgoing light intensity is modulated by the voltage applied to the LC cell. To recombine the two separated outgoing beams, we used a second but erected beam displacer, as depicted in Fig. 2. Continuous optical attenuation can be achieved by varying the frequency or amplitude of the applied voltage. The preferred approach is to keep the same voltage while switching the frequency. Relatively fast rise and decay times can be obtained because an electric field is present in both switching states. Increasing the bias voltage is favorable for shortening the rise and decay times. However, a high voltage driver is costly. Thus it is in our

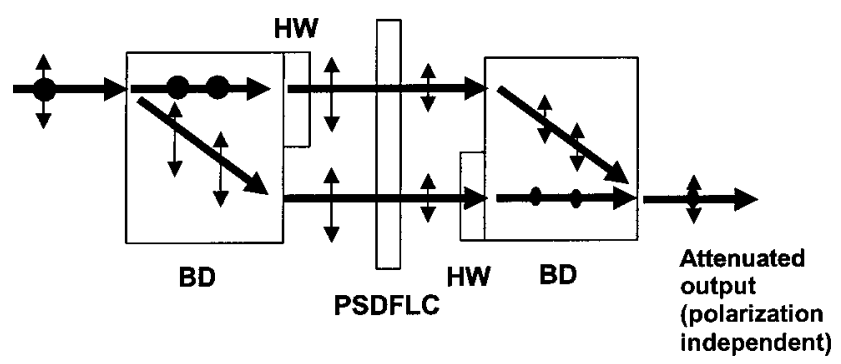

Fig. 2. Polarization-independent PSDFLC-based VOA; HW, halfwave plate; $\mathrm{BD}$, beam displacer; $\lambda=1.55 \mu \mathrm{m}$.

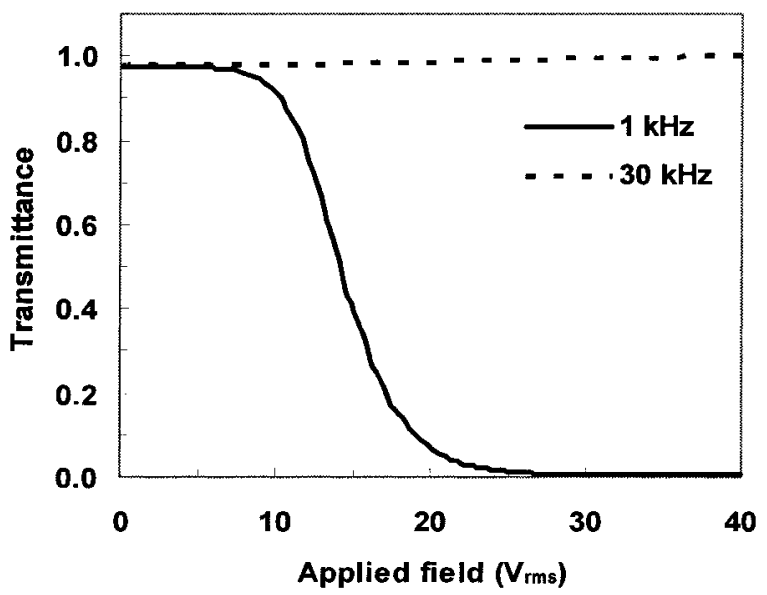

Fig. 3. Voltage-dependent transmittance of a PSDFLC-based VOA with $\lambda=1.55 \mu \mathrm{m}$.

interest to keep the operating voltage as low as possible.

\section{Experiment}

To fabricate the PSDFLC cell, we mixed 4 wt. \% of a rodlike monomer (M1) to a homemade dualfrequency mixture, designated as DFLC-25. The physical properties of DFLC-25 mixture are summarized as follows: birefringence $\Delta n=0.24$ at $\lambda=1.55 \mu \mathrm{m}$, dielectric anisotropy $\Delta \varepsilon=+5$ at $f=1 \mathrm{kHz}, \Delta \varepsilon=$ -4 at $f=30 \mathrm{kHz}$, and the crossover frequency is $f_{c}$ $=6 \mathrm{kHz}$ at $\mathrm{T}=25^{\circ} \mathrm{C}$. The LC-monomer mixture was injected into an empty homogeneous cell with gap $d=16 \mu \mathrm{m}$ and was then exposed to UV light $\lambda$ $\sim 365 \mathrm{~nm}$ at intensity $I \sim 6 \mathrm{~mW} / \mathrm{cm}^{2}$ for $\sim 30$ min at an elevated temperature, followed by $4 \mathrm{~h}$ at room temperature $\left(25^{\circ} \mathrm{C}\right)$. After the mixture was cured, the crossover frequency of the PSDFLC cell was found to increase from 6 to $8 \mathrm{kHz}$.

To characterize the PSDFLC's performance, a tunable laser (Ando AQ4321D) at $\lambda=1.55 \mu \mathrm{m}$ was used as the light source. Figure 3 depicts the voltagedependent transmittance measured by a computercontrolled LabVIEW system. At $f=1 \mathrm{kHz}$, the optical threshold voltage of the PSDFLC $\left(\mathrm{V}_{\text {th }} \sim 8.5 \mathrm{~V}_{\mathrm{rms}}\right)$ is much higher than that of the pure DFLC because of the polymer network effect. ${ }^{5}$ As the applied voltage increases, light scattering gradually increases so that the transmittance decreases. The photodetector was set at $7 \mathrm{~cm}$ behind the second beam displacer, which corresponds to $1.2^{\circ}$ acceptance angle. At a voltage of $24 \mathrm{~V}_{\mathrm{rms}}$, a good dark state is achieved and the measured dynamic range reaches $31 \mathrm{~dB}$. In an actual fiber-to-fiber system, the dynamic range should be higher than $31 \mathrm{~dB}$ because of the smaller acceptance angle. The large dynamic range originates from two mechanisms: the enhanced light scattering from micrometer-sized domains and the large index mismatch between the DFLC-25 mixture and the polymer networks. To obtain gray levels, we simply vary the frequency of the bias voltage. For example, in Fig. 2 , if we bias the VOA at $24 \mathrm{~V}_{\text {rms }}$ and increase the 


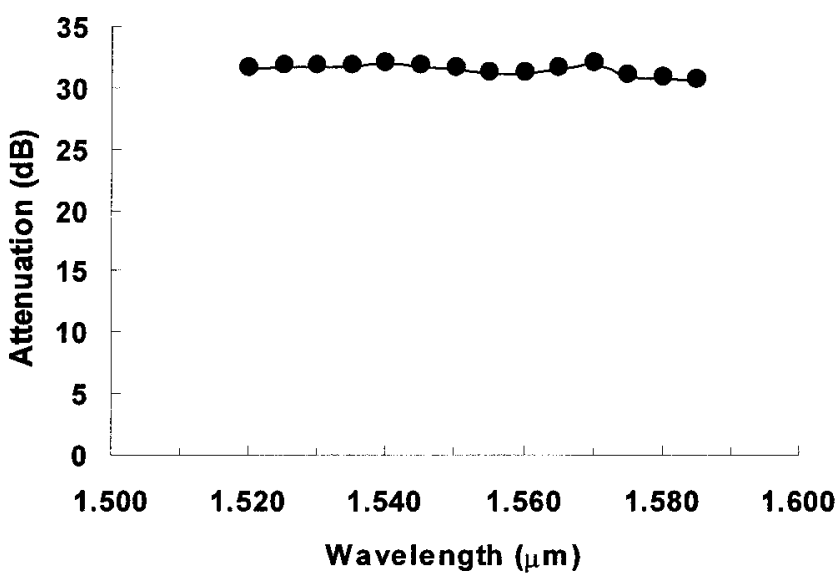

Fig. 4. Wavelength-dependent attenuation ratio of the PSDFLC VOA.

frequency from 1 to $8 \mathrm{kHz}$, various gray levels can be obtained.

The dashed line in Fig. 3 represents the measured transmittance by using a high-frequency electric field. For a homogeneous cell at $f=30 \mathrm{kHz}$, the effective dipole of the LC directors is parallel to the electric field. Such an electric field cannot reorient the LC molecules so that the transmittance is independent of the voltage.

For fiber-optic applications, a VOA is desired to have a broad bandwidth over the whole spectral range defined by the International Telecommunication Union. To investigate the wavelength dependency, we used an Ando amplified spontaneous emission light source $(\lambda=1525$ to $1575 \mathrm{~nm})$ and an optical spectrum analyzer. Figure 4 shows the wavelength-dependent optical attenuation of the PSDFLC VOA. The attenuation ratio remains relatively flat in the entire C-band. This is because the microdomain sizes are comparable with the wavelength.

Polarization-dependent loss (PDL) is another important parameter for fiber-optic application. Figure 5 plots the wavelength-dependent PDL of the VOA measured at the normal-on state. The variation is

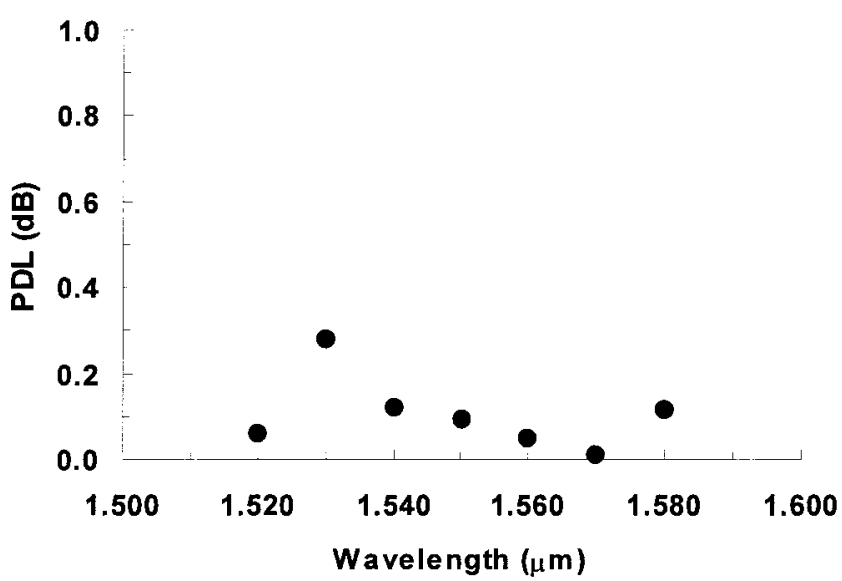

Fig. 5. Measured polarization-dependent loss (PDL) of the PSDFLC VOA with $d=16 \mu \mathrm{m}$.

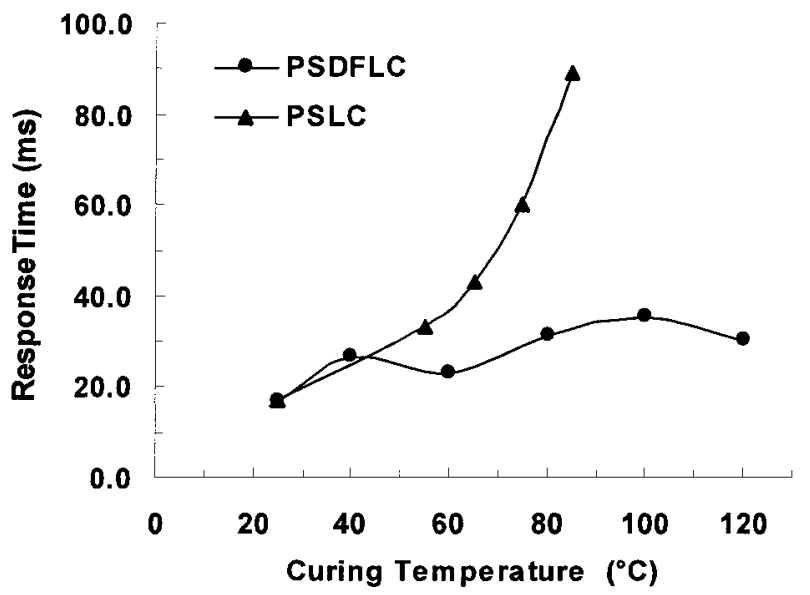

Fig. 6. Curing-temperature-dependent response time of PSDFLC (circles) and PSLC (triangles) cells.

approximately $0.1-0.2 \mathrm{~dB}$. The PDL increases as the attenuation increases. The PDL at $15 \mathrm{~dB}$ attenuation is approximately $0.5-0.6 \mathrm{~dB}$. On the basis of the data shown in Figs. 4 and 5, the PSDFLC exhibits promising properties for VOA applications.

\section{Discussion}

To obtain a wider dynamic range of a PSDFLC device, we could increase the cell gap, LC birefringence, and UV curing temperature. Light scattering increases as the cell gap increases. However, a thicker cell gap leads to a slower response time and a higher operating voltage. High-birefringence liquid crystals help to increase the dynamic range owing to their larger refractive-index mismatch, but their viscosity is increased because of their elongated molecular structures. Furthermore, higher viscosity increases the response time.

Curing temperature affects the domain size, dynamic range, and response time of a PSLC. ${ }^{13}$ Figure 6 shows the relation between the curing temperature and the measured total response (rise-and-fall) time. As the curing temperature increases, the response time of a regular PSLC cell increases dramatically. This is because the domain sizes increase with increasing curing temperature. In contrast, the PSDFLC cell is inert to the curing temperature; its response time is kept at $\sim 30 \mathrm{~ms}$ (rise time, $\sim 17 \mathrm{~ms}$; decay time, $\sim 13 \mathrm{~ms}$ ) when the curing temperature increases from $25{ }^{\circ} \mathrm{C}$ to $120{ }^{\circ} \mathrm{C}$. This is because the rise and decay times of the PSDFLC cell are driven by the applied low- and high-frequency electric fields. Therefore fast rise and decay times can always be achieved, regardless of the curing temperature. A high curing temperature plays a key role in enhancing the dynamic range. Thus we can use a high curing temperature to achieve a large dynamic range without sacrificing the response time.

In Fig. 6, the PSLC data represent a reflective scattering-type VOA by using a non-DFLC material. ${ }^{14}$ If the same LC layer thickness were used for a transmissive PSLC device, the dynamic range would 
be lower than $15 \mathrm{~dB}$ at $\lambda=1.55 \mu \mathrm{m}$ at each curing temperature. In our transmissive PSDFLC VOA, the dynamic range remains at $\sim 30 \mathrm{~dB}$ in the $60-120^{\circ} \mathrm{C}$ curing-temperature range. Moreover, the transmissive mode has a much simpler optical setup than the reflective mode and still preserves a high dynamic range and fast response time.

To further improve the PSDFLC VOA's response time, one could use the overdrive voltage method $^{15,16}$ or use a high-birefringence, low-viscosity DFLC mixture. The overdrive method is used to shorten the rise time by applying a short but high voltage to kick the LC molecules to the desired tilt angle, whereas the undershoot method is particularly useful for reducing the gray-scale response time. Although the overdrive and undershoot methods could lead to submillisecond response times, the required voltage is $\sim 100 \mathrm{~V}_{\mathrm{rms}}$.

The insertion loss of the PSDFLC cell is approximately $-3 \mathrm{~dB}$ owing to the quadruple passes of the uncoated glass substrates and the initial scattering effect in the transparent state. To reduce the insertion loss, we recommend that the LC cell be antireflection coated. In addition, by choosing a proper polymer material to match the refractive index of the LC material, one can further reduce the insertion loss. The hysteresis of the PSDFLC cells is relatively small at a high curing temperature. For example, the PSDFLC sample cured at $120{ }^{\circ} \mathrm{C}$ has a hysteresis of less than $0.1 \mathrm{~V}_{\text {rms }}$.

\section{Conclusion}

We demonstrate a transmissive VOA by using a polymer-stabilized dual-frequency liquid crystal (PSDFLC). By using the high-birefringence, dualfrequency material and polymer networks, we obtain a high-dynamic-range and fast-response-time device. In addition, the response time does not increase with increasing curing temperature. Therefore we could choose a higher curing temperature to achieve a larger dynamic range without sacrificing the response time. The PSDFLC approach can be extended to other scattering-type devices used in photonic applications.

This research is supported by the Air Force Office for Scientific Research under contract F49620-011-0377.

\section{References}

1. R. A. M. Hikmet, "Electrically induced light scattering from anisotropic gels," J. Appl. Phys. 68, 4406-4412 (1990).

2. R. A. M. Hikmet and H. J. Boots, "Domain structure and switching behavior of anisotropic gels," Phys. Rev. E 51, 58245831 (1995).

3. S. T. Wu and D. K. Yang, Reflective Liquid Crystal Displays (Wiley, 2001).

4. H. Ren and S. T. Wu, "Anisotropic liquid crystal gels for switchable polarizers and displays," Appl. Phys. Lett. 81, 1432-1434 (2002).

5. Y. H. Fan, Y. H. Lin, H. Ren, S. Gauza, and S. T. Wu, "Fastresponse and scattering-free polymer network liquid crystals for infrared light modulators," Appl. Phys. Lett. 84, 1233-1235 (2004).

6. R. A. Soref and D. H. McMahon, "Total switching of unpolarized fiber light with a four-port electro-optic liquid-crystal device," Opt. Lett. 5, 147-149 (1980).

7. E. G. Hanson, "Polarization-independent liquid-crystal optical attenuator for fiber-optics applications," Appl. Opt. 21, 13421344 (1982).

8. K. Hirabayashi, M. Wada, and C. Amano, "Optical-fiber variable-attenuator arrays using polymer-network liquid crystal," IEEE Photon. Technol. Lett. 13, 487-489 (2001).

9. J. J. Pan, H. Wu, W. Wang, X. Qiu, and J. Jiang, "Temperature independent, accurate LC VOA through electric feedback control," in Proceedings of the 19th National Fiber Optics Engineers Conference (Telcordia, 2003), pp. 943-949.

10. C. Mao, M. Xu, W. Feng, T. Huang, K. Wu, and J. Wu, "Liquidcrystal applications in optical telecommunication," in Liquid Crystal Materials, Devices, and Applications IX, L. C. Chien, ed., Proc. SPIE 5003, 121-129 (2003).

11. H. K. Bucher, R. T. Klingbiel, and J. P. Van Meter, "Frequencyaddressed liquid crystal field effect," Appl. Phys. Lett. 25, 186188 (1974).

12. M. Schadt, "Low-frequency dielectric relaxation in nematics and dual-frequency addressing of field effects," Mol. Cryst. Liq. Cryst. 89, 77-92 (1982).

13. F. Du and S. T. Wu, "Curing temperature effects on liquid crystal gels," Appl. Phys. Lett. 83, 1310-1312 (2003).

14. F. Du, S. Gauza, and S. T. Wu, "Influence of curing temperature and high birefringence on the properties of polymerstabilized liquid crystals," Opt. Express 11, 2891-2896 (2003).

15. S. T. Wu and C. S. Wu, "High speed liquid crystal modulators using transient nematic effect," J. Appl. Phys. 65, 527-532 (1989).

16. S. T. Wu, "A nematic liquid crystal modulator with response time less than $100 \mu \mathrm{s}$ at room temperature," Appl. Phys. Lett. 57, 986-988 (1990). 